\title{
Prescribing for adolescents
}

\section{Melissa Kang}

Associate professor, Public Health, University of Technology Sydney

\section{Kiely Kim}

Medical educator, GP

Synergy, Sydney

\section{Keywords}

adolescent development, informed consent

Aust Prescr 2019;42:20-3 https://doi.org/10.18773/ austprescr.2019.004

\section{SUMMARY}

The process of prescribing changes as children move into adulthood. For some medicines, such as psychotropic drugs, safety and efficacy are less well understood in adolescents.

As adolescents mature they attain the capacity to consent to their own medical treatment. An assessment of their competency will need to take into account the nature of the treatment being proposed.

Parental involvement is usually beneficial particularly for adolescents with chronic or complex conditions, but increasing adolescent autonomy needs to be respected.

Adherence to treatment can be supported by understanding adolescent development and involving adolescents in management plans.

\section{Introduction}

The challenges in prescribing for adolescents fall into four main categories:

- medicine safety and efficacy, taking into account the physiology of puberty

- the increasing autonomy of the adolescent patient

- conscious or unconscious clinician bias regarding young people

- concerns about adherence.

Prescribing decisions will involve clinical judgement, the adolescent's preferences, parent's or carer's wishes and the medicolegal framework.

\section{Prescribing patterns for adolescent patients}

Adolescents are defined by the World Health Organization as those aged 10-19 years. There are very little up-to-date data on prescription rates specifically for the adolescent population. An analysis of general practice surveillance data from 1998 to 2005 found the most frequently prescribed drugs in this age group were antibiotics for respiratory infections, antidepressants for anxiety or depression, and bronchodilators and corticosteroids for asthma. ${ }^{1}$ Among 14-17-year-old females, contraception is the second most commonly managed problem. Less than two-thirds of contraceptive prescriptions in this age group are for contraception, with menstrual problems and acne accounting for the rest. ${ }^{2}$

\section{Safety and efficacy}

It is important not to view adolescents as 'mini adults' when it comes to prescribing, although in general, the more advanced an adolescent is in puberty, the more similar their body composition and organ functions are to adults. This makes pharmacokinetic considerations simpler than they are in children. ${ }^{3}$ Nevertheless, physiological phenomena such as growth plate fusion and bone mass accrual which occur during puberty have implications for some prescribing decisions. For example, long-term use of depo-medroxyprogesterone is associated with osteoporosis, and long-term oral corticosteroids have the potential to affect growth. It is also important for clinicians who may have been prescribing drugs such as antiepileptics for adolescents when they were children to consider their sexual maturation and the possibility of pregnancy. Issues such as sexuality should be raised in a sensitive and confidential manner.

In younger adolescents whose peak growth velocity is still underway, paediatric dose calculations may be needed for some drugs, especially those with significant adverse effects. While several classes of drugs are safe to use in adult doses for adolescents aged 12 years and over, many have not been studied in this population, including psychotropic drugs. ${ }^{4}$ Clinicians often prescribe medicines off label with decisions based on research from adults because of limited knowledge about dosing, efficacy and safety in adolescents. ${ }^{5}$

For antidepressants, current evidence shows a modest benefit for only one drug in this age group (fluoxetine). ${ }^{6}$ There are also concerns about the risk of harm. Mild to moderate depression should be treated with non-pharmacological therapy. Decision making regarding psychotropic drugs requires careful consideration. A thorough assessment of mental health symptoms, a comprehensive biopsychosocial assessment, ${ }^{7}$ and a risk and safety assessment are warranted. Drug therapy is more likely to be indicated 
in moderate to severe depression or anxiety, in conjunction with non-pharmacological therapy, ${ }^{6}$ and is a shared process with the adolescent (and parent or caregiver). Collaboration with others involved in care, such as a psychologist and school counsellor, is important, as well as considering the need for a psychiatric opinion.

\section{Capacity to consent to medical treatment}

Adolescents attain cognitive capacity for reasoning and abstract thinking that, under Australian law, can afford them the legal status of 'mature minor'. All jurisdictions in Australia allow for the Common Law test of 'Gillick competence' which recognises that an adolescent under the age of 18 may have the capacity to consent to medical treatment on their own behalf and without their parents' knowledge. ${ }^{8}$ Additional legislation in South Australia and New South Wales grants adolescents aged 16 years and over (rather than 18 years) the right to consent to their own treatment as adults. ${ }^{9}$

An assessment of the adolescent's competency needs to be assessed in each case on a continual basis, and should consider the maturity of the adolescent as well as the nature and the complexity of the treatment.

To be competent the adolescent should be able to understand:

- what the treatment is for and why it is necessary

- $\quad$ any treatment options or alternatives

- what the treatment involves

- likely effects and possible adverse effects

- $\quad$ seriousness of the treatment

- consequences of not treating.

Options including the benefits and harms of treatment versus those of non-drug treatment should be discussed. Asking the adolescent to explain their own understanding of this can be helpful. It should be noted however that adults also have misunderstandings about medicines this is not unique to adolescents. For example, a large household survey in the UK found substantial misinformation about antibiotics. ${ }^{10}$ If unsure of an adolescent's capacity to consent, it is important to seek advice from a clinician with expertise in this area. In very complex cases a medical defence organisation may be able to provide advice.

\section{Confidentiality}

If adolescents ask for their parents not to be informed about their prescription, it is important to enquire about this wish. The concept of safety is understood by adolescents and is the basis for explaining exceptions to confidentiality. Discussing the adolescent's safety regarding drug treatment, as well as their health problem as the initial and primary concern, can be a useful way to encourage more openness with the parents. It can be revealing as well as practical to discuss with an adolescent what would happen if a family member were to discover their medicines. This can be a 'reality check' as well as an opportunity to anticipate reactions. Importantly, even though an adolescent may not want to involve a parent initially, this can change and it is important to maintain an ongoing dialogue about this. It is essential also that the adolescent understands confidentiality and its limits, and that if there are concerns about safety with risk to themselves, or others, there would be a need to inform their parent or caregiver.

If there are circumstances where an adolescent decides not to inform their parents, the context behind this should be explored and the reasons documented by the prescriber, as well as documenting having offered to speak with a parent.

The shifting power dynamics in the adolescentparent-clinician triad is a challenge for many clinicians. Difficulties reported by GPs include, on the one hand, negotiating time alone with adolescents to facilitate engagement, while on the other hand, feeling that adolescents are not sufficiently responsible to manage their health alone. ${ }^{11}$ What is unknown is whether clinicians change their prescribing behaviour as a result of these difficulties. For example, there is no published research that has explored prescribing decisions on the basis of parental presence or absence during consultations, whether parental consent is sought when prescribing for minors, and how often and for which drugs, or whether competency assessments are made.

\section{Possible bias}

There is also a dearth of information about the influence of conscious or unconscious bias towards young people in prescribing decisions, although interesting evidence is emerging in the area of contraception. A study among young Australian women (18-23 years) found that they perceived that doctors provided incomplete information about, and limited their options for, contraception because they were young. ${ }^{12} \mathrm{~A}$ recent qualitative study involving young women (16-24 years) of culturally diverse backgrounds also suggested that clinicians may be selective about the contraception options they offer. ${ }^{13}$ The uptake of long-acting reversible contraceptives has been slow among Australian women, despite them being recommended as first-line options for nulliparous 
women in Australia, the USA and the UK.14 This may be because Australian women and clinicians are unfamiliar with long-acting reversible contraceptives, compared to the combined oral contraceptive pill. ${ }^{14}$ However, there is a need to understand whether clinicians are consciously 'gatekeeping' and if so what influences this. Young women have the right to be informed about the full range of contraception options.

\section{Adherence}

Adherence to treatment is another challenge when prescribing for adolescents, particularly for those with a chronic illness such as diabetes. Rather than make assumptions about non-adherence among adolescents, it is the role of clinicians to work with adolescents to optimise adherence just as they would with adults. Understanding adolescent brain development, and how this influences behaviour, risktaking and decision making, can inform approaches to self-management.

In adolescence, peers have an impact on the processing of social information, resulting in a heightened sensitivity to peer attitudes. Adolescents are also more likely than adults to seek out situations that are arousing, exciting or stressful and they have greater difficulty than adults in overriding 'high emotions'.15

Understanding neurodevelopmental phenomena can help clinicians as well as the designers of programs intended to support adolescents with chronic illness as they transition from paediatric to adult care. Shared decision making is a goal in the management of chronic health conditions and enhances adherence, as do strategies such as motivational interviewing and pharmacist support. ${ }^{16}$ As children move into adolescence, the process of shared decision making needs to be supported by clinicians in consultation with the patient and their parents or carers. Listening to the adolescent's concerns can identify previously unconsidered barriers that may be amenable to change, such as dosage regimens interfering with school timetables, or concerns about adverse effects based on (mis)information on the internet or among peers. After identifying these concerns, additional strategies may include:

- education - providing accurate information and repeating at regular intervals in a variety of formats, for example verbal, written information, websites, books

- organisational - simple dosing, once-daily, extended-release formulations, alarms on watches, reminders on phones

- behavioural and problem solving, for example pairing taking medicines with other wellestablished behaviours

- $\quad$ peer and family support

- motivational interviewing techniques.

Evaluation of adherence is important at regular intervals when the adolescent and family have an opportunity to ask questions and discuss any treatment-related concerns. ${ }^{17}$

\section{Conclusion}

Adolescents are a group who are in a dynamic process of acquiring autonomy in all areas of life, including health care and self-management. Clinicians play a vital role in supporting this process and engaging adolescents in a provider-patient relationship that can have a positive impact on their health. Challenges around medicine safety, medicolegal concerns, clinician awareness about treatment bias, navigating the shifting dynamics in the parent-patient-clinician relationship, and finding strategies for improving adherence and self-management can all be addressed by first understanding adolescent development. Assessing psychosocial as well as medical histories should be routine and helps to build an empathic and trusting relationship with adolescent patients. $<$

Conflict of interest: none declared

\section{REFERENCES}

1. Booth ML, Knox S, Kang M. Encounters between adolescents and general practice in Australia. J Paediatr Child Health 2008;44:699-705. https://doi.org/10.1111/ j.1440-1754.2008.01409.x

2. Harrison C, Charles J, Britt H. Contraception. Aust Fam Physician 2011;40:93.

3. O'Hara K. Paediatric pharmacokinetics and drug doses. Aust Prescr 2016;39:208-10. https://doi.org/10.18773/ austprescr.2016.071

4. World Health Organization. Promoting safety of medicines for children. France: WHO; 2007. http://apps.who.int/iris/ handle/10665/43697 [cited 2019 Jan 3]

5. Psychotropic. In: eTG complete [Internet]. Melbourne: Therapeutic Guidelines Limited; 2018. www.tg.org.au [cited 2019 Jan 3]

6. National Institute for Health and Care Excellence. Depression in children and young people: identification and management. Clinical guideline (CG28). Published 2005 Sep. Updated 2017 Sep. https://www.nice.org.uk/guidance/ cg28 [cited 2019 Jan 3].

7. Chown P, Kang M, Sanci L, Newnham V, Bennett DL. Adolescent health: enhancing the skills of general practitioners in caring for young people from culturally diverse backgrounds. GP resource kit. 2 nd ed. Sydney: NSW Centre for the Advancement of Adolescent Health and Transcultural Mental Health Centre; 2008. https://www.health.nsw.gov.au/kidsfamilies/youth/Pages/ GP-resource-kit.aspx [cited 2019 Jan 3] 
8. Kang M, Sanders J. Medicolegal issues in adolescent health care. In Kang M, Skinner SR, Sanci L, Sawyer S, editors. Youth health and adolescent medicine. Melbourne: IP Communications; 2013.

9. Bird S. Consent to medical treatment: the mature minor. Aust Fam Physician 2011;40:159-60.

10. McNulty CA, Boyle P, Nichols T, Clappison P, Davey P. Don't wear me out--the public's knowledge of and attitudes to antibiotic use. J Antimicrob Chemother 2007;59:727-38. https://doi.org/10.1093/jac/dkl558

11. Kang M, Bernard D, Booth M, Quine S, Alperstein G, Usherwood T, et al. Access to primary health care for Australian young people: service provider perspectives. Br J Gen Pract 2003;53:947-52.

12. Goldhammer DL, Fraser C, Wigginton B, Harris ML, Bateson D, Loxton D, et al. What do young Australian women want (when talking to doctors about contraception)? BMC Fam Pract 2017;18:35. https://doi.org/10.1186/ s12875-017-0616-2

\section{FURTHER READING}

Headspace. Clinical toolkit - Clinical tips: checklist before prescribing SSRIs in young people. Available at: https://headspace.org.au/health-professionals/clinical-toolkit/ anxiety/management [cited 2019 Jan 3]

Orygen, The National Centre of Excellence in Youth Mental Health. Clinical Practice Guide - Treating depression in young people: guidance, resources and tools for assessment and management. Melbourne: Orygen; 2017. www.orygen.org.au/ Education-Training/Resources-Training/Resources/Free/ Clinical-Practice/Treating-depression-in-yp [cited 2019 Jan 3]
13. Botfield JR, Newman CE, Kang M, Zwi AB. Talking to migrant and refugee young people about sexual health in general practice. Aust J Gen Pract 2018;47:564-9.

14. Temple-Smith M, Sanci L. LARCs as first-line contraception What can general practitioners advise young women? Aust Fam Physician 2017;46:710-5.

15. Patton GC, Sawyer SM, Santelli JS, Ross DA, Afifi R, Allen NB, et al. Our future: a Lancet commission on adolescent health and wellbeing. Lancet 2016;387:2423-78. https://doi.org/10.1016/S0140-6736(16)00579-1

16. Usherwood T. Encouraging adherence to long-term medication. Aust Prescr 2017;40:147-50. https://doi.org/ 10.18773/austprescr.2017.050

17. Taddeo D, Egedy M, Frappier JY. Adherence to treatment in adolescents. Paediatr Child Health 2008;13:19-24. https://doi.org/10.1093/pch/13.1.19
Orygen, The National Centre of Excellence in Youth Mental Health. Evidence Summary - Shared decision-making for mental health: what is the evidence? Melbourne: Orygen; 2015. www.orygen.org.au/Education-Training/Resources-Training/ Resources/Free/Evidence-Summaries/Shared-Decision-Making/ Orygen_Shared_Decision_Making?ext [cited 2019 Jan 3]

Trapeze: a supported leap into adult health. The Sydney Children's Hospitals Network. www.trapeze.org.au [cited 2019 Jan 3] 